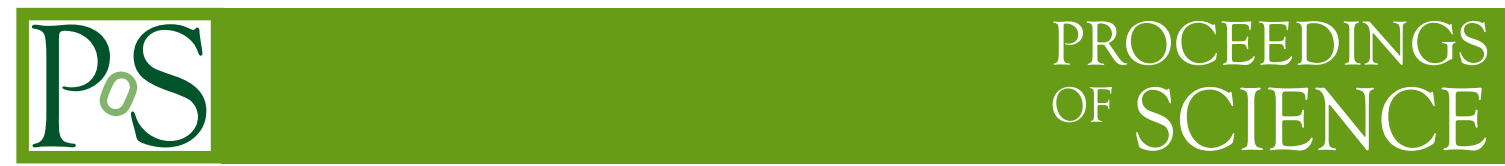

\title{
Jet correlations in forward production processes
}

\author{
F. Hautmann \\ Department of Theoretical Physics, University of Oxford, Oxford OXI 3NP \\ E-mail: hautmann@thphys.ox.ac.uk
}

\begin{abstract}
The production of hadronic jets in the forward region of pp collisions is affected by potentially large QCD logarithmic corrections both in the hard transverse momentum and in the large rapidity interval. The theoretical framework to resum consistently both kinds of logarithmic corrections to higher orders in perturbation theory is based on QCD high-energy factorization. We discuss Monte Carlo applications of this framework to final-state observables associated with production of one forward and one central jet, and present numerical results for forward-central angular correlations at the Large Hadron Collider.
\end{abstract}

35th International Conference of High Energy Physics

July 22-28, 2010

Paris, France 
The production of hadronic jets in the forward region of pp collisions forms a largely new area of experimental and theoretical activity at the Large Hadron Collider [1]. Forward jet production enters the LHC physics program in both new particle discovery processes (e.g., vector boson fusion channels [2] for Higgs boson searches) and new aspects of standard model physics (e.g., QCD at small $\mathrm{x}$ and its interplay with cosmic ray physics [3]).

The evaluation of QCD theoretical predictions for forward jets is made complex by the presence of multiple mass scales, possibly widely disparate from each other. This raises the question of whether fixed-order next-to-leading calculations reliably describe the production process or significant contributions arise which call for perturbative QCD resummations $[4,5,6]$ and/or corrections beyond single parton interaction $[7,8,9]$.

In the LHC forward jet kinematics, QCD logarithmic corrections in the large rapidity interval (of high-energy type) and in the hard transverse momentum (of collinear type) may both be quantitatively significant. The theoretical framework to sum consistently both kinds of logarithmic corrections to all perturbative orders is based on QCD high-energy factorization at fixed transverse momentum [5]. This factorization program is carried through in [6] for forward jet hadroproduction.

The multi-parton matrix elements computed in [6] factorize, in the high-energy limit, not only in the collinear emission region but also at finite angle. They can be used to take into account effects of coherence from multi-gluon emission away from small angles, which become important for correlations among jets across long separations in rapidity.

To achieve a fully exclusive description of the final states associated with forward jets, Ref. [10] employs a merging scheme based on high energy factorization $[5,6]$ to combine hard matrix elements and parton showering. The basic point is that, since radiative effects beyond leading order are encoded in off-shell matrix elements [6], one needs such a scheme to merge consistently the hard radiation from the short distance matrix element with the radiation from parton showering, by avoiding double counting. The other important point for the coupling to parton showers is that because in the forward kinematics one of the longitudinal momentum fractions $\mathrm{x}$ in the initial state becomes small, in order to take full account of multi-gluon emission coherence one needs to keep finite- $\mathrm{k}_{\mathrm{T}}$ terms in the initial-state parton branching [11]. In the numerical results [10] shown below this is done according to the shower algorithm of [12].

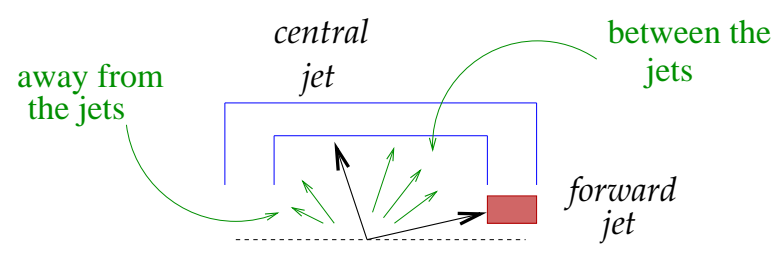

Figure 1: Production of forward and central jets.

At the LHC it is possible to measure events where jet transverse momenta $\mathrm{p}_{\mathrm{T}}>20 \mathrm{GeV}$ are produced several units of rapidity apart, $\Delta \eta \sim 3 \div 6$ [1]. Such multi-jet states can be relevant to new particle discovery processes as well as new aspects of standard model physics. Ref. [10] investigates correlations between forward and central jets (Fig. 1), in the framework outlined above, examining the effects of finite-angle gluon emission across the large rapidity interval. It compares these with effects of the multi-parton interaction corrections taken into account by [8]. 
The measurement of the azimuthal correlation of a central and forward jet provides a useful probe of how well QCD multiple-radiation are described. In [10] it is found that at the LHC, while the average of the azimuthal separation $\Delta \phi$ between the jets is not affected very much as a function of rapidity by finite-angle gluon emissions, the detailed shape of the $\Delta \phi$ distribution is. The cross section as a function of the azimuthal separation $\Delta \phi$ between central and forward jets reconstructed with the Siscone algorithm [13] $(R=0.4)$ is shown in Fig.2 [10] for different rapidity separations. The solid blue curve is the prediction based on implementing the factorization [6] in the parton-shower event generator [12] (CASCADE); the red and purple curves are the predictions based on calculations with collinear parton-showering [8] (PYTHIA), respectively including multiple interactions and without multiple interactions.

The decorrelation as a function of $\Delta \eta$ increases in CASCADE as well as in PYTHIA. In the low $E_{T}$ region (Fig. 2 (left)) the increase in decorrelation with increasing $\Delta \eta$ is significant. The cross section for jet separation up to $\Delta \eta<4$ is similar between CASCADE and PYTHIA with multiparton interactions, whereas a clear difference is seen to PYTHIA without multiparton interactions. However, at large $\Delta \eta>4$ the decorrelation predicted by CASCADE is significantly larger than the prediction from multiparton interactions. In the higher $E_{T}$ region (Fig. 2 (right)) CASCADE predicts everywhere a larger decorrelation. In this region, the influence of multiparton interactions in PYTHIA is small and the difference to CASCADE comes entirely from the different parton shower.
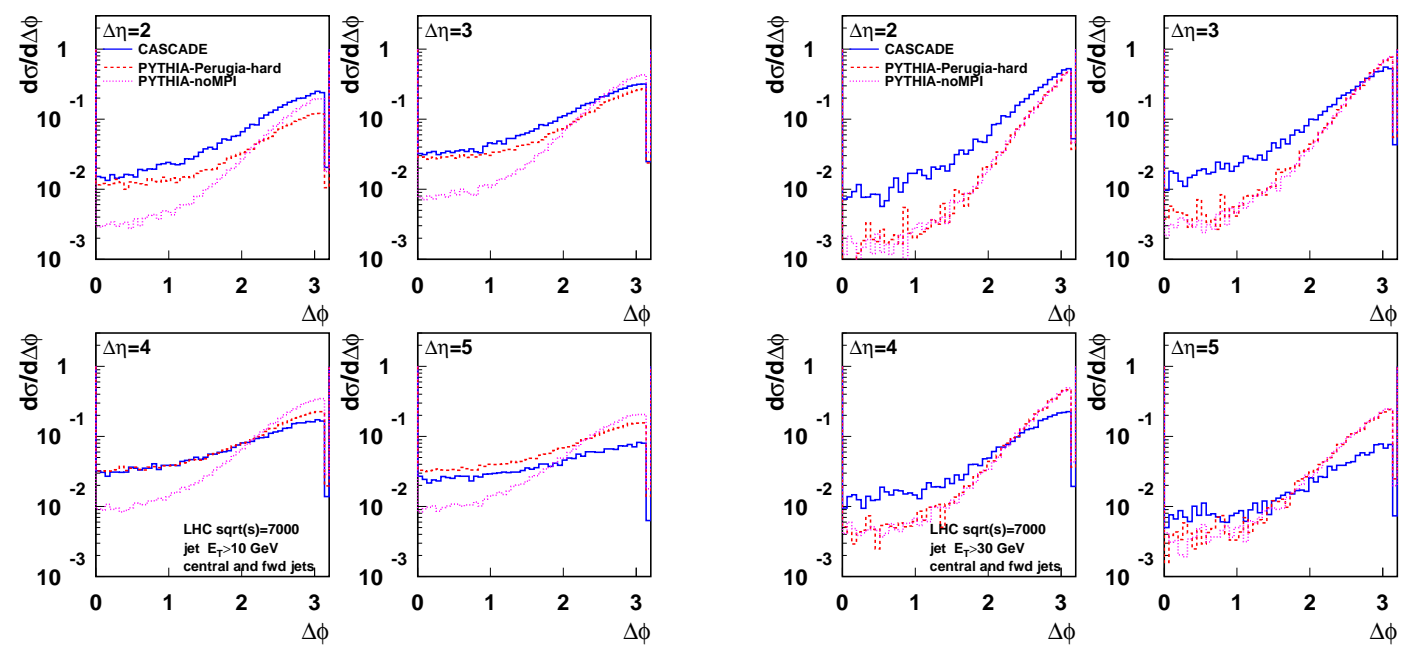

Figure 2: Cross section versus azimuthal separation $\Delta \phi$ between central and forward jet, at different rapidity separations $\Delta \eta$, for jets with transverse energy $E_{T}>10 \mathrm{GeV}$ (left) and $E_{T}>30 \mathrm{GeV}$ (right) [10].

Distinctive effects from the high-energy, noncollinear corrections to parton showers are also observed [10] in the $\Delta R=\sqrt{(\Delta \phi)^{2}+(\Delta \eta)^{2}}$ distribution, where $\Delta \phi=\phi_{j e t}-\phi_{\text {part }}\left(\Delta \eta=\eta_{\text {jet }}-\right.$ $\eta_{\text {part }}$ ) is the azimuthal (rapidity) difference between the jet and the corresponding parton from the matrix element. This distribution probes to what extent jets are dominated by hard partons in the matrix element or receive significant contributions from the parton shower. The large- $\Delta R$ region is found to be enhanced by noncollinear corrections, and while this signal can be mimicked by multi-parton interactions for low $E_{T}$ jets, this no longer applies as $E_{T}$ increases [10].

It is worth noting that many of the theoretical issues that underlie forward jet physics, from QCD resummations to parton-showering methods beyond leading order to potential effects of par- 
ton saturation [14], depend on the notion of transverse momentum dependent, or unintegrated, parton distribution functions (u-pdfs). (See [15] for recent reviews on this topic.) In the calculation above we take the high-energy definition of u-pdfs [5], namely, we rely on the fact that for small $\mathrm{x}$ u-pdfs can be defined gauge-invariantly (and can be related to the ordinary pdfs renormalized in the minimal subtraction scheme $\overline{\mathrm{MS}}$ [5]) by going to the high-energy pole in physical amplitudes. More general characterizations, valid over the whole phase space, are desirable, and currently the subject of much activity. Recent results in this area, see e.g. [16], are likely to eventually have a bearing on forward jet physics.

In conclusion, the analysis [10] shows that final states associated with forward jet production at the LHC get significant contributions from radiative corrections taking into account both large logarithms of rapidity and large logarithms of transverse momentum. Distinctive effects are seen by considering correlations between forward and central jets, e.g. azimuthal correlations. Phenomenological studies based on measurements of these correlations will be relevant for tests of initial state radiation and for the QCD tuning of Monte Carlo event generators. (For the counterpart of this in the case of central jet pairs see the first LHC measurements [17].) They can also be relevant to gain better control on the structure of the final states associated with heavy particle production (e.g., underlying jet activity in scalar boson production [18]).

This analysis can be extended to the case of forward and backward jets. It can thus serve to estimate the size of backgrounds from QCD radiation in Higgs searches from vector boson fusion channels [2].

Note that, besides LHC physics, forward jets are also relevant in the case of leptoproduction [19] for further analyses of ep Hera data [20] and for the physics program at the proposed future lepton facilities [21] (LHeC, EIC).

Studies of forward high- $\mathrm{p}_{\mathrm{T}}$ production, such as those discussed in this article, will be complemented at later stages of the LHC program by studies in other areas of forward physics employing near-beam proton taggers [22]. Both the high- $\mathrm{p}_{\mathrm{T}}$ and proton-tagging measurements can contribute to either standard-candle or discovery physics. In addition, both will provide inputs on (hard and soft) forward particle production that will serve for the modeling of high-energy air showers [3] in cosmic ray experiments.

Acknowledgments. The results presented in this article have been obtained in collaboration with M. Deak, H. Jung and K. Kutak.

\section{References}

[1] Z. Ajaltouni et al., arXiv:0903.3861 [hep-ph]; M. Grothe, arXiv:0901.0998 [hep-ex]; D. d'Enterria, arXiv:0911.1273 [hep-ex].

[2] K.J.C. Leney [on behalf of ATLAS Coll.], arXiv:0810.3144 [hep-ex]; M. Vazquez Acosta [on behalf of CMS Coll.], arXiv:0901.3098 [hep-ex].

[3] R. Engel, in Proc. 30th Int. Cosmic Ray Conf., ed. R. Caballero et al., vol. 6, p. 359 (2009).

[4] A.H. Mueller and H. Navelet, Nucl. Phys. B282 (1987) 727; C. Ewerz, L.H. Orr, W.J. Stirling and B.R. Webber, J. Phys. G26 (2000) 696. 
[5] S. Catani et al., Phys. Lett. B242 (1990) 97; Nucl. Phys. B366 (1991) 135; Phys. Lett. B307 (1993) 147; S. Catani and F. Hautmann, Phys. Lett. B315 (1993) 157; Nucl. Phys. B427 (1994) 475.

[6] M. Deak, F. Hautmann, H. Jung and K. Kutak, JHEP 0909 (2009) 121; arXiv:0908.1870 [hep-ph].

[7] P. Bartalini and L. Fanò (eds.), Proceedings 1st MPI Workshop (Perugia, 2008), DESY-PROC-2009-06; R.D. Field, in Proceedings 1st MPI Workshop (Perugia, 2008); M. Bähr, S. Gieseke and M. Seymour, JHEP 0807 (2008) 076.

[8] P.Z. Skands, arXiv:1005.3457 [hep-ph]; arXiv:0905.3418 [hep-ph], in Proceedings 1st MPI Workshop (Perugia, 2008), DESY-PROC-2009-06, eds. P. Bartalini and L. Fanò.

[9] B. Blok, Yu. Dokshitzer, L. Frankfurt and M. Strikman, arXiv:1009.2714; M. Strikman and W. Vogelsang, arXiv:1009.6123; T.C. Rogers and M. Strikman, Phys. Rev. D81 (2010) 016013; G. Calucci and D. Treleani, arXiv:1009.5881; S. Domdey, H.-J. Pirner and U.A. Wiedemann, Eur. Phys. J. C 65 (2010) 153; E. Maina, JHEP 0909 (2009) 081; JHEP 0904 (2009) 098; E.L. Berger, C.B. Jackson and G. Shaughnessy, Phys. Rev. D81 (2010) 014014; J.R. Gaunt and W.J. Stirling, JHEP 1003 (2010) 005; J.R. Gaunt, C.-H. Kom, A. Kulesza and W.J. Stirling, arXiv:1003.3953.

[10] M. Deak, F. Hautmann, H. Jung and K. Kutak, arXiv:1012.6037 [hep-ph].

[11] M. Ciafaloni, Nucl. Phys. B296 (1988) 49; S. Catani, F. Fiorani, and G. Marchesini, Nucl. Phys. B336 (1990) 18; G. Marchesini and B.R. Webber, Nucl. Phys. B386 (1992) 215; F. Hautmann and H. Jung, JHEP 0810 (2008) 113; arXiv:0804.1746 [hep-ph].

[12] H. Jung et al., arXiv:1008.0152 [hep-ph]; H. Jung, Comput. Phys. Commun. 143 (2002) 100.

[13] G.P. Salam and G. Soyez, JHEP 0705 (2007) 086; M. Cacciari et al., http://fastjet.fr.

[14] E. Avsar and A.M. Stasto, JHEP 1006 (2010) 112; E. Avsar and E. Iancu, Nucl. Phys. A 829 (2009) 31; E. Iancu, C. Marquet and G. Soyez, Nucl. Phys. A 780 (2006) 52.

[15] F. Hautmann, Acta Phys. Polon. B 40 (2009) 2139; F. Hautmann and H. Jung, Nucl. Phys. Proc. Suppl. 184 (2008) 64 [arXiv:0712.0568 [hep-ph]]; arXiv:0808.0873 [hep-ph].

[16] T. Becher and M. Neubert, arXiv:1007.4005 [hep-ph]; P.J. Mulders and T.C. Rogers, Phys. Rev. D81 (2010) 094006; A. Idilbi and I. Scimemi, arXiv:1009.2776 [hep-ph]; I. Cherednikov and N. Stefanis, Phys. Rev. D80 (2009) 054008; A. Bacchetta, D. Boer, M. Diehl and P.J. Mulders, JHEP 0808 (2008) 023; T.C. Rogers, Phys. Rev. D78 (2008) 074018; F. Hautmann, Phys. Lett. B655 (2007) 26; arXiv:0708.1319; J.C. Collins and F. Hautmann, JHEP 0103 (2001) 016; Phys. Lett. B472 (2000) 129.

[17] ATLAS Collaboration, arXiv:1009.5908 [hep-ex]; CMS Collaboration, arXiv:1010.0203 [hep-ex].

[18] M. Deak et al., arXiv:1006.5401 [hep-ph]; F. Hautmann, arXiv:0909.1240 [hep-ph]; Phys. Lett. B 535 (2002) 159; F. Hautmann, H. Jung and V. Pandis, arXiv:1011.6157.

[19] A.H. Mueller, Nucl. Phys. B Proc. Suppl. 18C (1990) 125; S. Catani, M. Ciafaloni and F. Hautmann, Nucl. Phys. B Proc. Suppl. 29A (1992) 182; J. Kwiecinski, A.D. Martin and P.J. Sutton, Phys. Rev. D46 (1992) 921; J. Bartels, A. De Roeck and M. Loewe, Z. Phys. C54 (1992) 635; W.K. Tang, Phys. Lett. B278 (1992) 363.

[20] A. Aktas et al., Eur. Phys. J. C46 (2006)27; S. Chekanov et al., Phys. Lett. B632 (2006)13; F.D. Aaron et al., Eur. Phys. J. C54 (2008) 389; A. Knutsson, LUNFD6-NFFL-7225-2007 (2007); L. Jönsson, AIP Conf. Proc. 828 (2006) 175.

[21] P. Laycock et al., "Future of DIS" summary report, in Proc. Workshop DIS 2010 (Florence, 2010).

[22] M.G. Albrow, T.D Coughlin and J.R. Forshaw, arXiv:1006.1289 [hep-ph]. 